\title{
A Photometric and Spectrographic Study of SX Phœnicis
}

\author{
Chulhee Kim ${ }^{1}$, D.H. McNamara ${ }^{2}$, Kent A. Feltz Jr. ${ }^{2}$, \\ C.G. Christensen ${ }^{2}$ \\ ${ }^{1}$ Department of Earth Science Education, Chonbuk National University, Korea, \\ ${ }^{2}$ Department of Physics and Astronomy, \\ Brigham Young University, Provo, UT 84602
}

\begin{abstract}
New simultaneous photometric $(u v b y \beta)$ and spectrographic observations of SX Phe are described. Analysis of the light variation frequency spectrum is performed and the oscillation modes are investigated. After separating the light curves and radial velocity curve, atmospheric and physical parameters corresponding to each different oscillation mode were determined. Intrinsic $(b-y), m_{1}$, and $c_{1}$ values used in conjunction with a model-atmosphere grid yield a mean effective temperature $\left\langle T_{\text {eff }}\right\rangle=7590 \mathrm{~K}$, a mean surface gravity, $<\log g>=4.02$, and $[\mathrm{Fe} / \mathrm{H}]=-0.47$ for the first period. The pulsation theory and stellar model sequences yield bolometric magnitude of $2^{m} \cdot 6$, mass of 1.4 $\mathcal{M}_{\odot}$ and age of 2.0 Gyrs. It was found that these atmospheric parameters are almost independent upon different oscillation mode. The radial-velocity data indicates a mean radial velocity of $-37 \mathrm{~km} / \mathrm{s}$ and a total velocity amplitude range of $38 \mathrm{~km} / \mathrm{s}$.
\end{abstract}

\section{Pulsating characteristics}

By adopting the generalized least squares method proposed by Vanicek (1971) for frequency analysis, we obtained that $f_{1}=18.1890(\mathrm{c} / \mathrm{d})$ and $f_{2}=23.3928(\mathrm{c} / \mathrm{d})$. Also sixteen frequencies which are harmonics or combiantions of two main frequencies are confirmed. The amplitude and phase for all photometric indices corresponding to each frequency were computed and light curves were well reproduced. We separated the light curves according to each of period. To do this, we subtracted the component of curve corresponding to the fundamental or first harmonic with all their overtones and all other combination terms. The radial velocity data was analyzed by a similar procedure and five frequencies were confirmed.

In order to identify the mode of pulsation, we applied the method developed by Watson (1988). To do this the amplitude ratio $\left(A_{B-V} / A_{V}\right)=0.351$ and 0.301 , and phase difference $\left(\phi_{B-V}-\phi_{V}\right)=6.02$ and 9.14 were computed for the first and second period by transforming $b-y$ to $B-V$ color indices. Then we applied model of $(7400$, $3.5)$ and $(8000,4.0)$ for $Q=0.03$ to identify the pulsation mode of SX Phe. We can see that the both modes are radial. We confirmed that this conclusion is supported by other methods. 


\section{Light curve analysis}

In order to discuss the variation in the physical parameters of SX Phe, we have sorted the photometric data by phase into 20 equally spaced bins around the light cycle corresponding to two periods for all $y, b-y, m_{1}, c_{1}$, and $\beta$ indices. Then normal points taken from smooth curves drawn through out the average points of each of the indices have been used in the data analysis. Intrinsic $(b-y)$ values were calculated with the aid of the Crawford calibration of $\mathrm{A}$ and $\mathrm{F}$ stars at normal points and we find an average color excess of $\langle E(b-y)\rangle=-0^{m} \cdot 016$ and $-0^{m} \cdot 009$ for the first and second period respectively. According to the early A-star calibration relating $[\mathrm{Fe} / \mathrm{H}]$ to $m_{1}$ by Crawford and Perry $(1976),[\mathrm{Fe} / \mathrm{H}]$ changes from -0.44 to -0.53 and -0.43 to -0.54 for the first and second period for all phase interval.

By interpolating the $(b-y)_{0}$ and $\left(c_{1}\right)_{0}$ values in a grid of model atmospheres computed by Kurucz corresponding to $[\mathrm{Fe} / \mathrm{H}]=-0.47$, it was confirmed that the effective temperature of SX Phe varies from $7210 \mathrm{~K}$ at light minimum to $8170 \mathrm{~K}$ at light maximum and the surface gravity varies from 3.89 to 4.27 for the first period.

We applied the Surface-Brightness Method to determine the radius and distance of SX Phe but we failed due to a large phase shift between radial displacement curve and angular diameter variation. However we obtained $1.5 R_{\odot}$ from the period - radius relation for dwarf cepheids. Also it was found that $R_{\min }=1.5 R_{\odot}$ from the theoretical $\left(\log R, \log P_{0}\right)$ diagram for $\delta$ Scuti variables by Andreasen, et al. (1983).

\section{Evolutionary status}

With the aid of the evolutionary models of VandenBerg for $Z=0.006$ and $Y=$ 0.25 we have calculated the evolutionary track in the $\log P-\log T_{\text {eff }}$ plane. It was confirmed that the mass of SX Phe is $1.4 \mathcal{M}_{\odot}$ and by referring to the models we find the age is $2.0 \mathrm{Gyr}$. Also by using $Q_{0}=0.033$ and $R_{\min }=1.5 R_{\odot}$, we obtained the pulsating mass of $1.2 \mathcal{M}_{\odot}$ for SX Phe. As a gravity mass, we obtained $1.5 \mathcal{M}_{\odot}$ by using our maximum surface gravity of 4.27 where the overestimation of 0.2 in $\log g$ for Stromgreen calibration was not taken into account.

According to the period-luminosity relation for fundamental mode pulsators, absolute magnitude of SX Phe is $2.8 \mathrm{mag}$ close to the overall absolute magnitude of 3.0 mag for Population II dwarf cepheids. However both are much different from $M_{v}=4.4 \mathrm{mag}$ calculated from parallax. Furthermore $\langle R\rangle=0.7 R_{\odot}$ can be obtained by utilizing Stefan-Boltzman law by taking our $T_{\text {eff }}=7590 \mathrm{~K}$ for the first period and $M_{b} \sim M_{v}=4^{m} .4$. All these inconsistent results are from uncertain parallax.

\section{References:}

Andreasen, G.K., Hejlesen, P.M., Peterson, J.O., 1983, A\&A, 121, 241

Vanick, P., 1971, ApSS, 12, 10

Watson, R.D., 1988, ApSS, 140, 255 